\title{
Detección de sintomatología de ansiedad social y factores asocia- dos en adolescentes de Motul, Yucatán, México
}

\author{
Alonso Humberto Marín-Ramírez ${ }^{1}$, German Jesús Martínez-Díaz ${ }^{1}$, José Manuel Ávila-Avilés ${ }^{2}$ \\ ${ }^{1}$ Facultad de Medicina, Universidad Autónoma de Yucatán, Mérida, México, ${ }^{2}$ Hospital Psiquiátrico de Yucatán, Mérida, \\ Yucatán
}

\section{RESUMEN}

Introducción. Existe una alta prevalencia de trastornos de ansiedad, y las fobias representan dentro de éstos, de 4.7 a $7.1 \%$ de los casos. Las fobias presentan un comienzo temprano, pudiendo ocasionar cronificación del cuadro ansioso y un aumento del riesgo de presentar comorbilidades psiquiátricas.

Objetivo. Determinar la prevalencia de sintomatología de ansiedad social y factores asociados en la población de estudiantes de secundaria de Motul, Yucatán.

Material y métodos. La población constó de 1,290 estudiantes que acuden a las escuelas secundarias en la ciudad de Motul. El tamaño de muestra se calculó mediante fórmula para muestreo estratificado, obteniéndose una muestra de 306 estudiantes. Los instrumentos utilizados fueron el Inventario de Fobia Social, el Test de Discriminación de Alcoholismo de Michigan, el Índice de Nivel socioeconómico de la AMAI, así como una encuesta para evaluar aspectos sociodemográficos.

Resultados. Se entrevistó a un total de 317 adolescentes, de los cuales 141 fueron mujeres. Se detectaron 49 alumnos con presencia de sintomatología de ansiedad social (15.4\%), de los cuales $34(69.3 \%)$ se encontraron en el rango de leve. $94 \%$ de los alumnos obtuvo resultado negativo en el test de Michigan. El nivel socioeconómico más prevalente fue el $\mathrm{D}+$. El valor más cercano a la significancia fue en el caso de sintomatología de ansiedad social por ocupación, con un valor de $\mathrm{p}=0.0532$.

Conclusiones. La prevalencia de sintomatología de ansiedad social en adolescentes fue de 15.4\%. No se encontró una relación de dependencia entre la sintomatología de ansiedad social y los factores evaluados.

Palabras clave: adolescente, prevalencia, ansiedad, trastornos fóbicos, alcoholismo.

\section{ABSTRACT \\ Detecting the symptomatology of social anxiety disorder}

Introduction. There is a high prevalence of anxiety disorders, and phobias represent about 4.7 to $7.1 \%$ of all anxiety disorders. Phobias start early in life and can lead to chronic anxiety disorder and an increased risks of psychiatric comorbidities.

Objective. To determine the prevalence of social anxiety disorder's symptoms and associated factors in the population of middle school students from Motul, Yucatán.

Material and methods. The population consisted Autor para correspondencia: Alonso Humberto Marín Ramírez, Calle 39 \#501 por 100 y Andador, Fraccionamiento Paseo de las Fuentes, Mérida,
Yucatán, México. CP: 97225 E-mail: amarinramirez@hotmail.com

Recibido: el 15 de octubre de 2014 Aceptado para publicación: el 5 de enero de 2015

Este documento está disponible en http://www.revbiomed.uady.mx/pdf/rb152614.pdf 
Marín-Ramírez et al.

of 1,290 students attending middle schools in Motul, Yucatán. The study sample size was calculated using the formula for stratified sampling, yielding a sample of 306 students. The instruments used were the Social Phobia Inventory, the Michigan Alcoholism Screening Test, the Index of Socioeconomic level of AMAI, and a survey to assess sociodemographic factors. Results. We interviewed a total of 317 students, of whom 141 were women. There were found 49 students with symptoms of social anxiety disorder (15.4\%), of which $34(69.3 \%)$ were in the mild range. $94 \%$ of students obtained negative results in the Michigan Alcoholism Screening Test. The most prevalent socioeconomic status was $\mathrm{D}+$. The closest value to the significance was social phobia symptoms versus occupation, with a $\mathrm{p}=0.0532$. Conclusions. The prevalence of social anxiety disorder's symptoms in adolescents was $15.4 \%$. No dependent relationship was found between social anxiety's symptoms and the evaluated factors.

Key words: adolescent, prevalence, anxiety, phobic disorders, alcoholism.

\section{INTRODUCCIÓN}

Datos sobre la prevalencia de los trastornos de ansiedad en México indican que estos padecimientos son los trastornos mentales más comunes; en la población mexicana alrededor de $14-15 \%$ de los individuos presentaba algún trastorno de ansiedad, y esta prevalencia llega hasta $18 \%$ en la población infantil y adolescente (1-3).

Los trastornos fóbicos representan de 4.7 a 7.1\% (4-6). La tasa de prevalencia varía según los criterios usados, pero empleando instrumentos de autoevaluación se ha encontrado una incidencia de $8.2 \%$ en población comunitaria adolescente (7).

A pesar de la amplia variabilidad epidemiológica reportada a nivel mundial con respecto a este padecimiento (8), en la Ciudad de México se encontró que los trastornos fóbicos son los más frecuentes en la población adolescente de ambos sexos, con una prevalencia de $10 \%$ en hombres y $12.4 \%$ en mujeres (9). Esta desigualdad, atribuida también a variaciones culturales, orienta a que, incluso dentro de un mismo país, se encuentren prevalencias diferentes según el área geográfica estudiada (9-11).

Estudios epidemiológicos señalan que la prevalencia de ansiedad social es mayor en mujeres, con una ocurrencia a lo largo de la vida de $15.5 \%$ frente a $11.1 \%$ en varones $(4,5)$.

El estatus y el rol social, la dependencia emocional y los ingresos económicos son justamente las características que explican que las mujeres sean mayormente expuestas y afectadas por el estrés social. El estatus de pareja también se asocia con la presencia de psicopatología en general y ansiedad social en particular. Las personas que tienen una pareja estable son más sanas física y mentalmente, en comparación con aquellas que viven solas (12).

La ansiedad social presenta una edad de inicio alrededor de los 11-16 años, aunque puede darse desde los 7 u 8 años, e incluso a edades tan tempranas como los 5 y tan avanzadas como los 35 años $(10,11)$. Este comienzo temprano ocasiona una afección en el desarrollo del adolescente, pudiendo derivar en cronificación del cuadro ansioso, pérdida de la calidad de vida, aumento del riesgo de consumo de sustancias tóxicas y de presentar otros cuadros mentales $(4,5,13,14)$.

En lo referente a las sustancias tóxicas, estas cobran importancia, especialmente en lo que respecta al consumo de alcohol. E1 alcoholismo puede preceder al trastorno de ansiedad, o puede ser consecuencia del mismo, ya que los adolescentes pueden llegar al consumo de sustancias como un intento por controlar su estado ansioso. Pese a que actualmente la evidencia de la efectividad de la medicación para estas dos afecciones no es concluyente, su detección oportuna es indispensable para lograr

\section{Revista Biomédica}


Detección de ansiedad social y factores asociados

implementar un tratamiento multidisciplinario (15-19).

Hasta en 70 a 90\% de los casos, la ansiedad social se complica por la presencia de estas condiciones, estimándose una comorbilidad de hasta 40-50\% con depresión mayor. En la práctica clínica es común que el médico reconozca estos otros trastornos antes que la ansiedad social $(7,8)$.

Aunado a esto, los trastornos fóbicos tienen la particularidad de que, en muchas ocasiones, son clínicamente subestimados por el médico de atención primaria, e incluso, por médicos especialistas (10) debido a que no se cuenta con el instrumento para su detección o porque el paciente puede considerarla normal.

La principal característica de la fobia social es un miedo persistente y acusado ante una amplia variedad de situaciones sociales; las personas afectadas presentan un miedo excesivo de ser evaluadas negativamente. Es característico que la ansiedad vaya ligada al estímulo, y cuando el paciente se ve forzado o es sorprendido por la situación fóbica experimenta una preocupación constante por la posibilidad de que aquellas situaciones resulten embarazosas $(4,5)$.

La exposición a estas situaciones suele generar respuestas psicofisiológicas de ansiedad, tales como sentir un nudo en la garganta, sensación de desmayo, dolores de estómago, llanto, tartamudeo o paralización $(4,5,7)$. Aunado a la sintomatología ansiosa, el individuo puede presentar síntomas somáticos, que van desde ruborización hasta crisis de angustia. Aunque se ha sugerido que los niños con trastorno de ansiedad social pueden tener una disfunción autonómica en la que presenten mayores niveles basales de estrés y una recuperación más lenta después del evento estresante, esto no se ha demostrado en todos los estudios, incluso cuando se comparan con un grupo control de niños sin trastornos de ansiedad (20).

Asímismo, suelen presentarse síntomas cognitivos como tendencia a autoevaluarse negativamente, hipersusceptibilidad a la crítica y baja autoestima, tendencia a temer las evaluaciones indirectas, o mostrar pobres habilidades sociales $(5,21)$. Se ha descrito que los niños con trastorno de ansiedad social tienen un mayor nivel de déficit de comunicación social, y que incluso en algunos casos éste déficit podría ser la causa subyacente al trastorno ansioso (22).

Actualmente, el DSM-5 presenta cambios con respecto a la edición previa en lo que concierne a la fobia social. El término ha sido cambiado por el de "Trastorno de ansiedad social", el cual refleja un nuevo y más amplio entendimiento de la enfermedad en una variedad de situaciones sociales. Con respecto a la duración de los síntomas, en el DSM-5 la duración de seis o más meses incluye también a los adultos. Anteriormente era necesario que el individuo afectado reconociera que su respuesta era excesiva y sin razón, pero actualmente el nuevo criterio permite al clínico tomar una decisión con respecto a esto (23).

En Yucatán, la Secretaría de Salud y el IMSS cuentan con programas de Salud Mental y del Adolescente, respectivamente, que están encargados de detectar casos de adicciones, violencia familiar, depresión y trastornos de la alimentación, mas no se cuenta con un programa para la detección de trastornos de ansiedad ni trastornos fóbicos. Incluso, no se cuenta con normas mexicanas que regulen la detección, valoración y tratamiento de estos padecimientos.

La poca información con que se cuenta en Yucatán sobre la epidemiología y los factores asociados a la ansiedad social contribuyen a la falta de detección de este padecimiento con la consecuente perpetuación del mismo y el aumento del riesgo de presentar los efectos crónicos que caracterizan a este trastorno.

El presente estudio tiene como objetivo detectar sintomatología de ansiedad social y los factores asociados a la misma, en una población de estudiantes de secundaria de Motul, Yucatán, México. 
Marín-Ramírez et al.

\section{MATERIAL Y MÉTODOS}

Se realizó un estudio descriptivo, observacional, prospectivo y transversal; la población incluyó a 1,290 estudiantes que acuden a las tres escuelas secundarias en la ciudad de Motul, Yucatán. El tamaño de muestra de 306 alumnos se obtuvo mediante una fórmula para muestreo estratificado, con una $\mathrm{pi}=0.50$, en cada uno de los estratos, y un nivel de confianza de 95\%. Las variables estudiadas fueron edad, género, nivel socioeconómico, sintomatología de ansiedad social, diagnóstico de enfermedad mental previa, estado civil, contar con hijos, ocupación, consumo de alcohol.

Para la recolección de datos se utilizaron los siguientes instrumentos: El Inventario de Fobia Social (SPIN), el Test de Discriminación del Alcoholismo de Michigan (MAST), el Índice de Nivel socioeconómico de la AMAI, y una encuesta que evaluó los factores sociodemográficos.

El Inventario de Fobia Social es una escala de autoevaluación, validada en población de adolescentes de habla hispana (24), que consiste en 17 reactivos con un formato Likert de cinco puntos; cada pregunta se califica de 0 a 4 , con un total de 68 puntos. Se han establecido puntos de corte para diferenciar entre los grados de la sintomatología: a) Puntuación $\leq 25$ : Sin síntomas atribuibles a fobia social; b) Puntuación 26 a 33: Sintomatología leve; c) Puntuación 34 a 40: Sintomatología Moderada; d) Puntuación $\geq 41$ : Síntomas intensos o severos. E1 SPIN ofrece una exactitud del $79 \%$ y se considera una prueba confiable, de consistencia interna, convergente y divergente. Entre otras ventajas se encuentra la seguridad para utilizarla en múltiples ocasiones, la brevedad de su aplicación, su sensibilidad a la fobia social, su facilidad para la puntuación y el hecho de que ha sido validad para poblaciones de adolescentes de habla hispana. Así mismo, los datos de esta escala indican un registro definido del umbral por lo cual uno puede separar la fobia social de otros trastornos psiquiátricos, y la fobia social de los controles no psiquiátricos $(8,25)$.
El MAST es un cuestionario que consiste en 25 preguntas de carácter dicotómico de respuesta sí o no, que tienen como objetivo la detección de problemas relacionados con el consumo de alcohol, así como la medición de la gravedad de los mismos. Muestra buena correlación con otros instrumentos similares para valoración del grado de abuso de alcohol. Utilizándose como instrumento clínico de detección, una puntuación entre 0 y 4 indica ausencia de alcoholismo, entre 5 y 6 sugiere posible alcoholismo y más de 7 indica probable alcoholismo (26).

El índice de Nivel Socioeconómico de la Asociación Mexicana de Agencias de Investigación de Mercados y Opinión Pública (AMAI) se considera el criterio estándar de clasificación de la industria de la investigación de mercados en México. El índice de nivel socioeconómico actual, conocido como Regla $13 \times 6$, clasifica a los hogares en seis niveles a partir de un árbol de asignaciones considerando 13 variables, divididas en una dimensión económica que es operacionalizada por la posesión de 12 bienes, y una dimensión social, que es operacionalizada por el nivel de estudio del jefe de familia. El nivel socioeconómico se clasifica en seis niveles, dependiendo de la puntuación obtenida: Nivel E: hasta 51 puntos, nivel D: entre 52 y 76 puntos, nivel D+: entre 77 y 133 puntos, nivel C: 134 y 170 puntos, nivel $\mathrm{C}+$ : entre 171 y 222 puntos, nivel A/B: más de 223 puntos (27).

Después de haber obtenido la autorización de los directores de las secundarias y el consentimiento informado de los alumnos; posteriormente, se realizó una reunión en la biblioteca con los encuestados, a los cuales se les entrego los 4 cuestionarios y se les indicó que respondieran de manera anónima, confindencial y veraz. Por otra parte, se realizó la estadística descriptiva para la presentación de los datos, según fuera, cualitativo o cuantitativo, y se determinó la relación entre las variables estudiadas y la

\section{Revista Biomédica}


Detección de ansiedad social y factores asociados

sintomatología de fobia social, mediante la distribución ji-cuadrada, y la obtención de riesgo para la cuantificación de la fuerza de la relación entre dos variables.

Para el análisis, se utilizó el programa estadístico Statgraphics Centurion.

Consideraciones éticas. Los alumnos mayores de edad entregaron cartas de consentimiento. En el caso de los alumnos menores de edad, se obtuvo el consentimiento informado por parte de los padres de los mismos. El proyecto fue aprobado por el Comité de Ética de la Facultad de Medicina de la Universidad Autónoma de Yucatán.

\section{RESULTADOS}

Se entrevistó un total de 317 adolescentes, de los cuales 179 fueron mujeres y 138 fueron hombres. La edad varió de 12 a 18 años, con $>60 \%$ entre los 13 y 14 años de edad. 97\% de los encuestados reportó su estado civil como soltero, habiendo ocho alumnos en unión libre, y únicamente 1 alumno casado. Solamente 4 alumnos (1\%) reportaron contar con hijos. Hubo una mayor prevalencia de alumnos que eran únicamente estudiantes (282), mientras que 11\% reportó tener como ocupación estudiar y trabajar. Una baja proporción (3.1\%) refirió haber recibido atención psicológica previa.

Se encontraron 49 casos con presencia de sintomatología de ansiedad social, lo cual representa $15.4 \%$ del total de la muestra. El mayor porcentaje de los alumnos que presentaron la sintomatología se encontraron en el rango de leve (34 alumnos). La mayoría de los alumnos obtuvo resultado negativo en el test de alcoholismo de Michigan, mientras que $6 \%$ obtuvo resultado de posible o probable a dicha condición. Se encontró que la mayor proporción de los alumnos se ubicó en el nivel socioeconómico D+ $(30.2 \%)$, C+ (27.1\%) y C (20.8\%). El nivel socioeconómico $\mathrm{E}$ fue el que menos alumnos tuvo.

Al realizar el cruce de variables, únicamente se encontró 1 alumno $(0.32 \%)$ con sintomatología leve y 1 con sintomatología moderada, con posible alcoholismo. Se encontró una distribución azarosa y sin patrón específico de los alumnos con sintomatología de ansiedad social con respecto al nivel socioeconómico. No se encontró predominio de sintomatología de ansiedad social de ningún grado en algún nivel socioeconómico específico. No hubo predominio de sintomatología de ansiedad social en ningún sexo.

De los 49 alumnos que presentaron ansiedad social, 64\% fueron femeninos y $36 \%$ fueron masculinos. Esta misma proporción se mantiene a través de todos los grados de sintomatología de ansiedad social, como se observa en el Cuadro 1. Se observó que la mayoría $(97 \%)$ de los pacientes que presentaron sintomatología de ansiedad social se encontraban solteros, aunque esto no tuvo significancia estadística. Ninguno de los cuatro alumnos que contaba con hijos presentó algún grado de sintomatología de ansiedad social. 89\% de los alumnos que presentaron sintomatología de ansiedad social fue estudiante. Esta proporción se repite también para cada uno de los grados de ansiedad en particular: sintomatología leve, 97\% estudiantes; moderada, 64\% estudiantes; sintomatología severa, $100 \%$ estudiantes.

Al investigar la relación de dependencia de sintomatología de ansiedad social con el resto

Cuadro 1

Frecuencia de sintomatología de fobia por Género

\begin{tabular}{lccc}
\hline & Femenino & Masculino & Total por fila \\
\hline Leve & 22 & 12 & 34 \\
& $(6.94 \%)$ & $(3.79 \%)$ & $(10.73 \%)$ \\
Moderada & 8 & 5 & 13 \\
& $(2.52 \%)$ & $(1.58 \%)$ & $(4.10 \%)$ \\
Severa & 1 & 1 & 2 \\
& $(0.32 \%)$ & $(0.32 \%)$ & $(0.63 \%)$ \\
Sin & 148 & 120 & 268 \\
sintomatología & $(46.69 \%)$ & $(37.85 \%)$ & $(84.54 \%)$ \\
Total por & 179 & 138 & 317 \\
columna & $(56.47 \%)$ & $(43.53 \%)$ & $(100.00 \%)$ \\
\hline
\end{tabular}


Marín Ramírez et al.

de las variables, resultó que en todas ellas los valores de $\mathrm{p}$ fueron mayores que 0.05 (Cuadro 2). El valor más cercano a la significancia fue en el caso de sintomatología de fobia social por ocupación, con un valor de $\mathrm{p}=0.0532$, con un mayor porcentaje de los pacientes con sintomatología de ansiedad social en el grupo de los alumnos que únicamente eran estudiantes.

\section{DISCUSIÓN}

El total de alumnos detectados con presencia de sintomatología de fobia social representa15.4\% del total de la muestra. Esta prevalencia se encuentra entre las más altas detectadas en la bibliografía, independientemente si los estudios utilizaron criterios diagnósticos del DSM-IV o autoinformes $(7,8)$.

Incluso cuando se compara con respecto al sexo, la prevalencia en este estudio (17.3\% de las mujeres y $13 \%$ de los hombres) es mayor que la reportada por Corina-Benjet en un estudio realizado en estudiantes de la Ciudad de México, en el que se reportó que la fobia social ocupa el segundo lugar entre los trastornos psiquiátricos en la población adolescente de ambos sexos, con una prevalencia de $12.4 \%$ en mujeres y de $10 \%$ en hombres (9).

Sin embargo, esto no se reflejó como una mayor prevalencia de sintomatología de ansiedad social en la población femenina $(\mathrm{p}=0.7345)$. Esta

Cuadro 2

Interferencia estadística: Pruebas de Independencia

\begin{tabular}{lccc}
\hline \multicolumn{1}{c}{ Factor } & Estadístico & G1 & Valor-P \\
\hline $\begin{array}{l}\text { Sintomatología de fobia por } \\
\text { alcoholismo }\end{array}$ & 3.062 & 6 & 0.8010 \\
$\begin{array}{l}\text { Sintomatología de fobia por } \\
\text { nivel de socioeconómico }\end{array}$ & 9.840 & 15 & 0.8297 \\
$\begin{array}{l}\text { Sintomatología de fobia por } \\
\text { sexo }\end{array}$ & 1.277 & 3 & 0.7345 \\
$\begin{array}{l}\text { Sintomatología de fobia por } \\
\text { estado civil }\end{array}$ & 2.538 & 6 & 0.8642 \\
$\begin{array}{l}\text { Sintomatología de fobia por } \\
\text { hijos }\end{array}$ & 0.741 & 3 & 0.8636 \\
$\begin{array}{l}\text { Sintomatología de fobia por } \\
\text { ocupación }\end{array}$ & 7.677 & 3 & 0.0532 \\
\hline
\end{tabular}

falta de significancia estadística con respecto a la ansiedad social y el sexo también se encontró en el estudio en el que Garcia-Lopez validó el SPIN en una población de adolescentes españoles (24).

A pesar de que la literatura indica que la ansiedad social puede coincidir con el alcoholismo $(4,24,28)$ en este estudio no se encontró significancia estadística al comparase los alumnos positivos en el test MAST con los positivos en el SPIN ( $p=0.8010)$.

Además de la posibilidad de que en realidad los alumnos no ingieran alcohol, pudo existir un posible sesgo en el que los alumnos, al estar expuestos a sus compañeros, no hubieran respondido de manera adecuada la encuesta, a pesar de que se les recalcó que sus resultados permanecerían anónimos. Asímismo, el test de Michigan es un test para adultos, que pudo haber presentado falsos negativos en la población encuestada en este estudio. Cabe recalcar que, como se describe en la literatura, también existe la posibilidad de que la sintomatología de ansiedad social pueda estar antecediendo al inicio del abuso de sustancias tóxicas.

A pesar de que se ha reportado que factores como el nivel socioeconómico, el rol y estatus social, la ocupación, y el estatus de pareja $(4,12,29)$, pueden ser factores relacionados con la presencia de ansiedad social, en este estudio no se encontró una relación estadísticamente significativa entre la presencia de sintomatología de ansiedad social y alguna de estas variables.

Con respecto al nivel socioeconómico y el estatus social, la explicación del rol poco satisfactorio y carente de recompensas y prestigio, así como el estar sometido a presión social (12), podría no encontrar lugar en los adolescentes encuestados, quienes a la edad de 13-14 años pertenecen al mismo estatus social, viven en una comunidad relativamente pequeña, y la mayoría no ejerce una profesión que les retribuya económicamente y les dé un lugar de prestigio en la comunidad que forman, que es prácticamente homogénea.

\section{Revista Biomédica}


Detección de ansiedad social y factores asociados

Con respecto a la relación entre ocupación y ansiedad social, a pesar de que no se encontró relación entre ambas variables, el valor de $p=0.0532$, coincide con lo reportado en la literatura, donde se menciona que, tener ingresos económicos propios, podría estar relacionado con una menor prevalencia de sintomatología de ansiedad social. La gente que tiene ingresos propios presenta una menor presión social al ubicarse en un estrato social más elevado, lo cual pudiera estar fungiendo como protector contra la ansiedad.

Por otra parte, es importante recalcar que a pesar de que la bibliografía menciona que las personas que tienen una pareja estable son más sanas física y mentalmente en comparación con aquellas que viven solas, en la práctica clínica con población adolescente, el estatus de pareja podría ejercer el efecto opuesto. La mayoría de los adolescentes que aún están en fase de desarrollo físico, psicológico y sexual, no suelen tener una pareja relativamente estable, lo cual en lugar de fungir como protector, provocaría incluso más estrés psicológico, que puede derivar en una mayor ansiedad en general.

El no haber encontrado en esta investigación un factor relacionado con la ansiedad social no descarta la existencia de otros factores o que, incluso, las variables encuestadas en este estudio puedan desarrollarse de manera tardía.

Existe una multitud de otras variables que pueden estar relacionadas con la ansiedad social, que van desde la obesidad (30) y el déficit de atención con hiperactividad, hasta las relacionadas con la vulnerabilidad genética y las que se asocian al efecto de las experiencias ambientales en el aprendizaje y el comportamiento del niño.

Desafortunadamente, no existen características personales o miedos específicos que nos permitan establecer con mayor certeza qué pacientes desarrollarán ansiedad social. Incluso aún debe establecerse en qué medida los reportes para investigar este tipo de ansiedad nos aportan información fidedigna para el diagnóstico, llegándose a sugerir que se debe contrastar el dato referido por el niño o adolescente, con el del padre del mismo, para obtener una mayor concordancia sobre las situaciones sociales que ocasionan ansiedad (31).

De igual importancia, es que en este estudio se pudo evidenciar el contraste entre el porcentaje de ansiedad social encontrado, $15.4 \%$, y el porcentaje de los alumnos que habían recibido atención psicológica previa, que fue únicamente de $3.1 \%$. Cabe recalcar que ninguno de los alumnos con resultado positivo a sintomatología de ansiedad social refirió haber recibido atención psicológica previa. Esto nos muestra que ciertamente los pacientes con ansiedad social no acuden al médico por su sintomatología, y cobra importancia debido a que, como se ha constatado, es el médico de atención primaria quien puede ejercer la mayor influencia para derivar a estos pacientes, recomendar el tratamiento farmacológico o la psicoterapia (32).

El día de hoy se desconoce el grado en que cada uno de los factores mencionados puede explicar la génesis del trastorno de ansiedad social. Se ha sugerido abordar los trastornos psicopatológicos desde un punto de vista jerárquico, donde las afecciones particulares se encuentren dentro de un rango de características psicopatológicas comunes, que puedan predisponer, mediante la internalización o externalización, a los diferentes trastornos mentales, entre los que se encontrarían el trastornos de ansiedad social y los trastornos por abuso de sustancias. Este enfoque tendría implicaciones nosológicas, etiológicas y clínicas, que permitiría entender la enfermedad mental desde un punto de vista más general (33).

Resulta necesario seguir investigando a fin de poder llegar a determinar el peso de los factores asociados en el contexto de un modelo explicativo de vulnerabilidad, inicio, desarrollo 
Marín-Ramírez et al.

y mantenimiento de este trastorno de la conducta y la personalidad.

\section{REFERENCIAS}

1. Medina-Mora ME, Borges G, Benjet C, Lara C, Berglund P. Psychiatric disorders in Mexico: lifetime prevalence in a nationally representative sample. BJPsych. 2007; (190): 521-8.

2. Martínez-Ortiz AL, Fernández-Aguirre CE, Navarro-Machuca IG, Martínez-Martínez MO. Guías clínicas del Hospital Psiquiátrico Infantil "Dr. Juan N. Navarro". Diagnóstico y manejo de los trastornos de ansiedad - Secretaría de Salud, México. 2008.

3. Virgen-Montelongo R, Lara-Zaragoza AC, Morales-Bonilla G, Villaseñor-Bayardo SJ. Los trastornos de ansiedad. Revista Digital Universitaria. [en línea] 2005 Noviembre. [Fecha de acceso Mayo 2014]. Disponible en: http://www.revista.unam.mx/ vol.6/num11/art109/nov_art109.pdf

4. Kaplan HI, Sadock BJ. Sinopsis de Psiquiatría. Ciencias de la conducta. Psiquiatría clínica. $10^{\circ}$ Edición. Editorial Panamericana; 2009.

5. Hales RE, Yudofsky SC. Tratado de psiquiatría clínica. $4^{\circ}$ Edición. Editorial Masson, 2004.

6. Gabbard, GO. Tratamientos de los trastornos psiquiátricos. $1^{\circ}$ edición. Editorial Ars Medica; 2008.

7. García-López LJ, Piqueras JA, Díaz-Castela MM, Inglés CJ. Trastorno de ansiedad social en la infancia y adolescencia: estado actual, avances recientes y líneas futuras. Behavioral Psychology / Psicología Conductual. 2008; 16(3): 501-33.

8. Rivera-Benitez C, Sánchez-Hernández VH. Sistema de Actualización Médica. Medicina Interna. $1^{\circ}$ edición. México: Intersistemas Editores; 1998.

9. Benjet C, Borges G, Medina-Mora ME, Méndez E, Fleiz C, Rojas E, et al. Diferencias de sexo en la prevalencia y severidad de trastornos psiquiátricos en adolescentes de la Ciudad de México. Salud Mental. 2009 Marzo-Abril; 32(2): 155-63.

10. Clinical Practice Guidelines for the Management of Anxiety Disorders. Can J Psychiatry. 2006 July; 51(Suppl 2): 35-41.

11. Kristensen $\mathbf{H}$, Torgersen $\mathrm{S}$. Social anxiety disorder in 11-12-year-old children. The efficacy of screening and issues in parent-child Agreement. Eur Child Adolesc Psychiatry. 2006; 15(3): 163-71.

12. Robles-García R, Espinosa-Flores RA, PadillaGutiérrez AC, Álvarez-Rojas MA, Páez-Agraz F. Ansiedad Social en Estudiantes Universitarios: Prevalencia y Variables Psicosociales Relacionadas. Psicología Iberoamericana. 2008 Julio-Diciembre;
16(2): 54-62.

13. Bados A. Fobia social. Universitat de Barcelona, Facultat de Psicologia, Departament de Personalitat, Avaluació i Tractament Psicològics. Enero 2009.

14. Programa de salud del adolescente y joven. Gobierno de Chile. Atención De Adolescentes Con Problemas De Salud Mental. Chile: Ministerio de salud; 2009.

15. Galbraith T, Heimberg RG, Wang S, Schneier FR, Blanco C. Comorbidity of Social Anxiety Disorder and Antisocial Personality Disorder in the National Epidemiological Survey on Alcohol and Related Conditions (NESARC). J Anxiety Disord. 2014 January; 28(1): 57-66.

16. Saiz-Martinez PA, Jimenez-Trevino L, Diaz-Mesa EM, Garcia-Portilla-Gonzalez MP, MarinaGonzalez P, Al-Halabi S, et al. Patología dual en trastornos de ansiedad: recomendaciones en el tratamiento farmacológico. Adicciones. 2014; 26(3):254-274.

17. The British Psychological Society and The Royal College of Psychiatrists. Social anxiety disorder. The nice guideline on recognition, assessment and treatment. London; 2013.

18. Ipser JC, Wilson D, Akindipe TO, Sager C, Stein DJ. Pharmacotherapy for anxiety and comorbid alcohol use disorders. Cochrane Database Syst Rev. 2015 January; 20(1). Disponible en: http:// onlinelibrary.wiley.com/doi/10.1002/14651858. CD007505.pub2/pdf/abstract

19. Smith JP, Randall CI. Anxiety and Alcohol Use Disorders: Comorbidity and Treatment Considerations. Alcohol Research: Current Reviews. 2012; 34(4): 414-31.

20. Alkozei A, Creswell C, Cooper PJ, Allen J. Autonomic arousal in childhood anxiety disorders: Associations with state anxiety and social anxiety disorder. J Affect Disord. [en línea] 2014 Dec [Fecha de acceso 21 enero 2015]. Disponible en: http://www. jad-journal.com/article/S0165-0327(14)00780-0/pdf

21. American Psychiatric Association Of Washington. DSM-IV-Manual diagnóstico y estadístico de los trastornos mentales. $4^{\circ}$ edición. Barcelona: Editorial Masson; 1995.

22. Halls G, Cooper PJ, Creswell C. Social communication deficits: Specific associations with Social Anxiety Disorder. J Affect Disord. [en línea] 2014 Oct [Fecha de acceso 21 enero 2015]. Disponible en: http://www.jad-journal.com/article/S01650327(14)00594-1/pdf

23. American Psychiatric Association. Diagnostic and statistical manual of mental disorders. 5th ed. 
Detección de ansiedad social y factores asociados

Arlington, VA: American Psychiatric Publishing; 2013.

24. Garcia-Lopez LJ, Bermejo RM, Hidalgo MD. The Social Phobia Inventory: Screening and Cross-Cultural Validation in Spanish Adolescents. The Spanish Journal of Psychology. 2010; 13(2): 970-80.

25. Letamendi AM, Chavira DA, Stein MB. Issues in the Assessment of Social Phobia: A Review. Isr J Psychiatry Relat Sci. 2009; 46(1): 13-24.

26. Universidad Complutense de Madrid. Proyecto de apoyo a la evaluación psicológica clínica. Ficha técnica. Banco de instrumentos psicométricos. [Recursos electrónicos] 2005. [Consultado en Mayo 2014]. Disponible en: http://pendientedemigracion. ucm.es/info/psclinic/evaluacion/Proyecto $\% 20$ Apoyo $\% 20$ EPC $\% 202006 /$ INSTRUMENTOS $\% 20$ E V A L U A C I O N / T R A S T O R N O S \% 20 RELACIONADOS\%20CON\%20SUSTANCIAS/ TEST\%20DE\%20DISCRIMINACION\%20DEL \%20

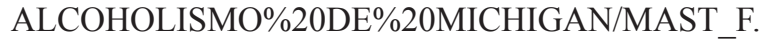
PDF

27. INEGI. Nivel socioeconómico AMAI. [En línea] Mayo, 2008. [Consultado en Mayo 2014]. Disponible en: http://www.inegi.org.mx/rne/docs/Pdfs/Mesa4/20/ HeribertoLopez.pdf

28. Zubeidat I, Fernández Parra A, Sierra JC, Salinas
JM. Evaluación de factores asociados a la ansiedad social y a otras psicopatologías en adolescentes. Salud Mental. 2008 Mayo-Junio; 31(3):189-96.

29. Caraveo-Anduaga JJ, Colmenares E. Prevalencia de los trastornos de ansiedad fóbica en la población adulta de la ciudad de México. Salud Mental. 2000 Octubre; 23(5): 10-9.

30. Calderón C, Forns M, Varea V. Implicación de la ansiedad y la depresión en los trastornos de alimentación de jóvenes con obesidad. Nutr Hosp. 2010; 25(4): 641-7.

31. Puliafico AC, Comer JS, Kendall PC. Social Phobia in Youth: The Diagnostic Utility of Feared Social Situations. Psychological Assessment. 2007; 19(1): $152-8$.

32. Weisberg RB, Dyck I, Culpepper L, Keller MB. Tratamiento psiquiátrico en pacientes de atención primaria con trastornos de ansiedad: comparación de la asistencia recibida de profesionales de atención primaria y de psiquiatras. Am J Psychiatry (Ed Esp). 2007 Mayo; 10(5): 298-304

33. Blanco C, Wall MM, He JP, Krueger RF, Olfson $\mathbf{M}$, Jin CJ, et al. Space of Common Psychiatric Disorders in Adolescents: Comorbidity Structure and Individual Latent Liabilities. J Am Acad Child Adolesc Psychiatry. 2015; 54(1):45-52 